\title{
Aesthetics, Art, Evolution
}

\author{
Jon McCormack \\ Centre for Electronic Media Art \\ Monash University, Caulfield East, Australia \\ Jon.McCormack@monash .edu \\ http://jonmccormack.info
}

\begin{abstract}
This paper discusses issues in evolutionary art related to Art Theory and Aesthetics with a view to better understanding how they might contribute to both research and practice. Aesthetics is a term often used in evolutionary art, but is regularly used with conflicting or naïve understandings. A selective history of evolutionary art as art is provided, with an examination of some art theories from within the field. A brief review of aesthetics as studied in philosophy and art theory follows. It is proposed that evolutionary art needs to resolve some important conflicts and be clearer about what what it means by terms like "art" and "aesthetics". Finally some possibilities for how to resolve these conflicts are described.
\end{abstract}

Keywords: Evolutionary Art, Art Theory, Aesthetics.

\section{Introduction}

The moment an artist accepts the effort of describing how he works, he reduces his way of working to that description. He strips it of its embedding into a living body and being.

-Frieder Nake [31, p.92]

Research in evolutionary art (from here referred to as $E A$ ) traditionally focuses on technical approaches to developing systems that generate or analyse artefacts which are considered on an "aesthetic" basis. While the evolutionary computing and technical aspects of EA come from an established scientific field, very little art theory has been used to inform research in EA. The "art" in EA seems to be largely taken for granted and is passed with little commentary or analysis from within the field. This appears to be a serious deficiency. How can a field of enquiry that claims to involve itself in art do so without an intellectual engagement in art itself?

This paper provides some basic explanation about art and aesthetics from philosophy and art theory. I will argue that an understanding of art and aesthetic theory would benefit EA, even if it is only to acknowledge that EA is not really concerned with Art (in a contemporary sense) at all. Almost every EA research paper mentions "aesthetics" as something that is fundamentally driving the research. Yet, what kind of aesthetics is implied, or objectified, is unusually 
inferred only by association with the mandatory visual examples that research papers provide. So, what does EA mean when it speaks about aesthetics? And how does it relate to contemporary understandings in art and philosophy?

I am expressly addressing evolutionary visual art in this paper. Music and sound arts form an important and significant component of EvoMUSART and certainly have much in common from technical perspectives. However, I have deliberately not included a discussion on music and sound art as I think the issues are often very different and would force unhelpful generalisations.

\section{Evolutionary Art}

This paper is addresses issues relating to evolutionary art, art theory and aesthetic theory originating in philosophy and art. Implied by its title, EA is also apparently concerned with art, but examination of the literature shows that what is meant by "art" has received little critical attention or explanation from within the field itself. It does at first seem unusual that an area of investigation which aims to create or understand art has provided scant examination of the subject of its investigation. What kind of "art" is EA really concerned with?

\subsection{EA and Art}

The field of EA is generally agreed to have begun with the work of British artist William Latham, who in 1988 first evolved sculptural forms by hand and then later, with the assistance of programmer Steven Todd, transferred the process to a computer at IBM research laboratories in Winchester, UK [40]. Shortly following, Karl Sims (a researcher at Thinking Machines Corporation) produced a series of seminal papers and short animations of evolved images and procedural 3D forms. Latham was trained as an artist, having developed his practice at the Royal College of Art in the 1980s. Sims came from a technical background in computer science and biology, and like Latham was assisted by a number of programmers while he developed his work at Thinking Machines. Richard Dawkins' 1986 book The Blind Watchmaker [9] included a software program to evolve biomorphs (two-dimensional stick drawings) using what is now known as the Interactive Genetic Algorithm or Aesthetic Selection. Dawkins' application demonstrated how designs could emerge without the teleological direction of a designer, with Dawkins himself claiming "Nothing in my biologist's intuition, nothing in my 20 years' experience of programming computers, and nothing in my wildest dreams, prepared me for what actually emerged on the screen" (p. 59). Blind Watchmaker was not intended as a work of art, even though it understandably inspired many evolutionary artists that followed.

In the early days of EA, the "Art World" peripherally showed some interest in this new kind of art. Exhibitions at established museums and art galleries, such as the Centre Georges Pompidou in Paris [2], alongside venues established for exhibiting electronic and computer art, such as Ars Electronica [18] in Austria and SIGGRAPH in the USA briefly made evolutionary and Artificial Life (A-life) 
$\operatorname{art}^{1}$ their artistic and intellectual focus. Latham and Sims were joined by artists such as Christa Sommerer and Laurent Mignonneau, the author, Steven Rooke, Nik Gaffney and Jane Prophet, to name just a (selective) few. These artists developed their works in the early to mid 1990s. Texts such as Mitchell Whitelaw's Metacreation, [41] published in 2004, but largely based on his $\mathrm{PhD}$ thesis completed in 2001, provided important critical theory that connected the technical advances of EA to broader concerns in culture, society, and art. However, the impact of this theory on the broader art community is debatable.

It is fair to say that the art world quickly grew tired of evolutionary and A-life art only a few years after it began and has had little or no interest since. There are a number of possible explanations for this, including the diffusion of evolutionary issues into other areas, such as robotic art, bio-art, and media installation along with emerging tensions between technology-based art and the art mainstream. Art of the late twentieth and early twenty-first century tends to be always distracted, never favouring any topic for very long anyway, with the notable exception of an introspective obsession with art itself.

In broader terms, there was a reaction to the virtual and computer art of the 1990s with its dependency on virtual representations and glittering screenbased computer graphics. Generative art based on computer software also kept its internal mechanisms (i.e. the code itself) hidden, ${ }^{2}$ choosing only to display the products of the code graphically or sonically. In contrast, software art made code itself the performative medium, inviting its audience to consider code itself as artistic material that was open to critique and questioning [1].

Hal Foster's The Return of the Real [15], again addressing the concept of mimeses in art, heralded a return to materiality along with embodied and social concerns in art. Over the ensuing decade, computer graphics quickly embedded itself into mainstream culture, making it and the culture that surrounds it (games, for example) the subject of critical and social analysis for art itself, as opposed to the inert and benign approach reflected in early EA.

A compelling explanation for the lack of interest in EA as art is summed up by Jussi Parikka [34]:

... if one looks at several of the art pieces made with genetic algorithms, one gets quickly a feeling of not "nature at work" but a Designer that after a while starts to repeat himself. There seems to be a teleology anyhow incorporated into the supposed forces of nature expressed in genetic algorithms.

Parikka is also troubled by a "vague characterisation of art" in a number of EA papers and is critical of the references to Art exclusively as a "subjective element", where art-making is understood in pre-modernist terms as a craft, but with digital tools. Parikka is also critical of the narrow understanding of

\footnotetext{
${ }^{1}$ A-life art commonly used evolutionary techniques which involved agent-based simulation or generative techniques, and so is considered here as a form of EA.

2 This isn't the case with live coding, for example, however live coding is most commonly associated with music performance, not visual art.
} 
aesthetics, where the emphasis is on surface appearances that are "interesting" or "beautiful". This issue is further explored in Section 3.

While EA made little impact in the art world, it developed and maintained a stronger link with design and computer science. Takagi's survey paper demonstrated a wide variety of applications for the IGA [39]. But it also highlighted the two major problems: user fatigue and the limited benefit of the IGA for experienced or advanced users. Nevertheless, IGAs made their way into a number of commercial music and visual design software systems (such as the "Brainstorm" feature found in Adobe After Effects [7]). Lewis also undertook an extensive survey of EA, highlighting the significant and varied contribution it has made in art and design since its inception [25]. However he notes ominously in conclusion "Methods for identifying and measuring progress in aesthetic research, as always, remain uncertain".

Like Parikka, a recent paper by Philip Galanter suggests that after 20 years of active practice "a vague feeling of disappointment surrounds evolutionary art" [16]. He suggests a problem with innovation in EA lies in representation and the ability of a system to exhibit - as nature does - multiple levels of emergence (a dynamic hierarchy), a view similar to that proposed in $[30,27,28]$, and also similarly does not advance any practical means to achieve it in software.

Galanter also offers two main contributions to EA art theory. The first is the concept of effective complexity, appropriated from physicist Murray Gell-Mann [17] and vaguely reminiscent of Birkhoff's famous proposal of aesthetic measure [6]. Galanter sees effective complexity as a valuable means to classify art and even provides formal graphs locating a number of algorithmic techniques on an idealised complexity curve, with "Genetic Systems and A-Life" at the pinnacle. There are, however, a number of problems with this approach. Firstly, unlike Birkhoff and despite being able to place specific algorithms on a graph, Galanter provides no method for measuring this complexity, either for a generative system or what it generates. As effective complexity isn't defined for the systems and artefacts it seeks to describe, it has no way of being evaluated, or worse, measured. Certainly, images people find interesting lie somewhere between nothing and noise, ${ }^{3}$ but the relative positioning of one technique in relation to another misunderstands the fact that a number of generative techniques are Turing complete, i.e. they are able to simulate a universal Turing machine and hence, run any computable program, including the systems at other points on Galanter's graph. For example, a Turing machine can be built from a cellular automata running the Game of Life [5], or from an L-system grammar or semi-Thue system [35].

The second difficulty with effective complexity "theory" is that, even if effective complexity could be evaluated for EA systems, it simply provides a 1-dimensional classification that says little about the works themselves. The rational for using effective complexity is that it provides an indication of the non-random information in the system that is generating the artwork, but as with all simple measures, it says nothing about the value or semantics of that

\footnotetext{
${ }^{3}$ Although the extremes have been popular at times in art too.
} 
information, nor the semiology. Moreover, randomness is often an evocative and meaningful element in art [24].

Galanter's second contribution is one of "truth to process": evolution is not teleological, so fitness-driven EA presents a "contradiction" because it evolves for a specific purpose (presumably the purpose of personal aesthetics). Galanter requires the evolutionary process to be true to natural evolution if it is to be beautiful. The connection between truth and beauty goes back a long way in art (see Section 3.3) and relates fundamentally to mimeses, discussed in Section 3.

A computer program that seeks to model or mimic evolution must necessarily abstract and simplify, which requires value-judgments as to what the important aspects of the model are: what we choose to incorporate into the model and what we choose to ignore. How we model a specific feature is in turn subject to representational and semantic interpretation. Such issues are well explored in simulation science, where models require validation with the system or phenomena they are modelling [33]. A simulation, $S$, can be tested for validation against a system $P$, if the mapping, $h$, is homomorphic, i.e. $h: P \rightarrow S$. Art in general requires no such verification or validation - a fundamental difference to the sciences and perhaps illustrative of the difficulties faced in scientific approaches to making art.

The problem of requiring a system to be "true" to what it seeks to emulate requires us to know what kind of truth we are after, if it is a homomorphic truth, then what differentiates EA from scientific simulation?

Johnson, addressing the call for more art theory in EA proposed in [27], considers a series of possibilities for future EA research [21]. These include techniques such as generational memory, scaffolding, connotation and web search in the context of fitness evaluation for EA. Johnson's approach is practically oriented, bringing in ideas from human creative process for example, rather than being tied to a faithful reproduction of biological evolution. He also suggests EA systems should engage with the "outside world", rather than being closed simulations. Web searches could be used to obtain a list of connotations to make make the work "about something without this 'something' being directly represented" (such an approach has been successfully undertaken in The Painting Fool, developed by Colton and his group [8]). But this approach plays to the criticisms of O'Hear ([32], discussed in the next section) that any such computer generated art can only be parasitically meaningful, because it is derived from existing material with no communicable understanding of that material. Of course, one may readily ignore this distinction and still interpret an implied communication where none really exists, just as natural patterns or events may readily invoke meaning in a conscious observer.

\subsection{EA and Philosophy}

An important goal in EA is to generate, using evolution, things that might be in some sense considered creative or artistic. The basis of this evaluation is generally only considered in folk terms ( $I$ don't know much about art but I know what I like...). However, ignoring issues of evaluation for a moment, let 
us consider this proposition in analytic terms. Taken to an extreme, EA raises the possibility of a computer creating art, that is, in a certain sense being an autonomous artist. ${ }^{4}$ Some philosophers have argued that computers can never create art as a matter of principle, because art "in the full sense is based in human experience" and requires a communication between artist and audience drawn from that shared experience [32]. ${ }^{5}$ However, while a machine cannot originate anything from direct human experience, this does not render it impossible in principle to understand enough about human perception and experience so as to allow meaningful communication. So, in principle (if not in deed), EA may have a role in practical philosophy.

Indeed, evolutionary simulation in software has proven a useful philosophical tool (see, e.g. [10,11]) allowing philosophers to conduct thought experiments in silico. This actually seems a promising area for EA to explore: areas such as cultural evolution or consideration of the art world as a generative system being two topical examples ([36] and [19] are good examples).

\section{Aesthetics}

\subsection{A Selective History of Aesthetics}

Aesthetics is an extensively studied field in philosophy and art, its modern meaning originates from Alexander Baumgarten's 1735 master's thesis [4] and his unfinished book Aesthetica. Baumgarten's hope was to bring a rationalist understanding of the science of perception to the critical judgement of perfection (i.e. beauty) in poetry. Hence, aesthetics is concerned with human perception and sensory experience. However currently the term is used not only in this sense of philosophical enquiry, but with a variety of other meanings. For example, we may talk about a "minimalist aesthetic" in relation to a design or the "aesthetic experience" of hang gliding over a snowy, windswept mountain range (explored further in Section 3.2).

There is also a tradition in philosophical aesthetics originating before Baumgarten that is concerned with issues relating to beauty, truth and morality as being "ultimate values" that are pursued for their own sake. Plato, for example, saw art and poetry as mimetic, judging them as only poor imitations of ideal truths. Thomas Aquinas sought to shift the transcendental beauty from the senses to the intellect, bringing it inline with theistic doctrines that associate perfection and truth with the divine. Such views persisted for centuries (and to some extent still today permeate certain artistic discourses) until the arrival of philosophers such as Nietzsche who sought to understand human experience without recourse to the divine. His writings are also interesting to EA, as he discusses the way tools influence cognition - writing on a typewriter is different than writing with a word processor, for example. Ideally, EA as art changes our

\footnotetext{
${ }^{4}$ This issue is further discussed in [29].

${ }^{5}$ I assume O'Hear would not be a fan of animal art or young children's art as Art, despite numerous exhibitions, critiques and regular publicity in the popular press.
} 
understanding of what art is or can be, but as a tool the computer influences how we think about "making art". One also suspects that a background in computer science has an even greater influence.

While the relationships between beauty, representation and truth have changed significantly over the centuries in art, they remain important issues in any discussion of art, including EA.

Mimesis is another reoccurring theme in art, and of particular interest to EA, as evolutionary art is mimetic at a variety of levels (e.g. as a process that imitates nature, in what is produced, and through representation of mathematical functions, for example). The idea of replicating naturalistic effects in painting came to the fore in renaissance aesthetics, where painters were concerned with a truthful representation of what they saw. Roughly corresponding with the mathematical formalisation of perspective projections and with progressive advances in paint technologies [3], artists' skills developed in portraying the "real" in art. However, any art acting as a "mirror of nature" - as famously advocated by Leonardo - still requires interpretation and ordering from the artist. As discussed in Section 2.1, one cannot have a mirror of nature in simulation either, because by definition, a simulation of something is not the thing itself.

Kant's Critique of Judgement [22], first published in 1790, firmly located aesthetics with the study of sensory and emotional experience, replacing associations with metaphysical ideals and truths with a more subjective appreciation routed in experience. Kant was still aware of the interplay between phenomenological experience and cognition. How much is the appreciation of beauty determined through sensory experience and how much from cognition and intellect? The latter raises more pertinent issues in art as it involves conscious experience (and hence unconscious experience) bringing the entire nature of experience into play and the role of culture and society informing individual identity, and hence, their judgments on beauty.

Kant also developed the idea of "disinterested pleasure", that is being interested in something as a means in itself, rather than for material gain, benefit to one's self or one's kin. From an evolutionary perspective, the concept of disinterest may at first seem problematic. Why attend to something if it offers no benefit other than in the act of interest itself? What is the evolutionary advantage of such behaviour? Here evolutionary psychology and the role of evolutionary forces such as sexual selection come into play, a mechanism that has been useful for EA.

\subsection{Modern Aesthetics}

Koren differentiates ten different meanings of the term "aesthetics" in modern culture $^{6}[23]$. They are:

1. The superficial appearance of things - the way they look, feel or sound on the surface (suggesting pure sensory experience);

${ }^{6}$ Koren defines these as "natural meanings", that exist beyond dictionary definitions, in the sense in which people in modern societies actually use the term. 
2. A particular style or sensibility - perceptual cohesive organisation or traits used to form groupings, e.g. "modernist", "baroque", "minimalist", "dubstep", "EvoArt", etc.;

3. A synonym for taste - the ability to recognise and identify artistic and stylistic features in things;

4. In the philosophy of art - the concept of disinterested experience, objective vs. subjective, experience vs. cognition, etc.;

5. A coherent statement of opinion or belief relating to the underlying principles of art or beauty;

6. A synonym for "artistic";

7. A synonym for beauty or the beautiful;

8. The services of a profession devoted to the beautification of the human body;

9. A cognitive mode that considers the sensory and emotive qualities of phenomena and things (as opposed to the direct sensory experience of those phenomena or things). The inner, subjective experience of thoughts, such as the "poetic resonance of a name given to a newly discovered type of subatomic particle." According to Koren, this implies that all of reality is essentially aesthetic phenomenon;

10. A language used by a community involved in art, design, or similar endeavours.

EA regularly uses the term "aesthetics" and this seems to be in the sense of a number of Koren's meanings (most commonly 1, 2, 3, 6 and 7). As a basic principle, it would be helpful for researchers to clarify what they mean by aesthetics in their own EA research. Considering the other interpretations would be additionally helpful. Moreover, aesthetics and art are not the same thing. EA often sees art only in terms of artefact and object, defined by surface appearance with the aim of making this appearance interesting or beautiful to researchers and their peers (or at least implicitly justifying its interest value without explicit reference to any formal aesthetic theory).

\subsection{Beauty}

... scientists are clear about a function for art. It is there, is it not, to show us the intrinsic beauty of the world?... [but] they are surprised to learn that 'beauty' is a word used sparingly by artists who want to do more than simply record or illustrate objects and ideas.

—Siân Ede [14, p.47]

What then is beauty specifically? Scruton lists six properties of the beautiful: beauty is pleasurable; it is relative; it is a reason in itself for attention; it is the subject of judgements of taste and these judgements are about properties held outside the self; judgements of taste require sensory experience (i.e. they cannot be conferred from another linguistically, for example) [38]. This last feature is interesting because it implies there is critical information in sensation which cannot be proxied through other media. This might ultimately be determined 
by the physicality of both the thing being perceived and the physicality of perception itself, in addition to the physics of the intervening medium. To judge something beautiful is to bring that thing to one's attention, and the "truly" beautiful evokes an emotional response in the viewer or listener.

Of course, we might consider other means of understanding beauty, from the perspective of evolutionary psychology or neuroscience for example. Humphrey sees humans drawn to beauty as a dog is drawn to saccharine - there is an innate desire to find beauty in certain constructs of likeness tempered with difference [20]. Humphrey sees aesthetics as a biological predisposition of humans and animals to seek classification of structure in the world around them. Beautiful structures facilitate classification since they provide evidence of possible taxonomies in ways that are easy to understand. Such an approach sees analogies with more recent information theoretic understandings [37].

\section{Does Art Matter to EA?}

Does understanding aesthetic theory and art really help EA? After all, dealing with the intangible and unmeasurable, with recourse to metaphysics and seemingly impenetrable continental philosophy doesn't necessarily sit comfortably with a scientific, or even scientistic approach to understanding. I would argue that if EA seeks to be accepted as relevant art, it must first understand the dialogues in art regarding representation, menesis, beauty and truth (to name just a few). These discourses are centuries old and they do have something useful to offer, even if your goal is not explicitly artistic. Further, if EA is interested in participating in contemporary art, this can only be achieved by considering art as a process and on-going social exchange, rather than an exclusively scientific study of objects and their appearance. This is problematic for a conference such as EvoMUSART, which largely (but to its credit, not exclusively) focuses on peer-reviewed research papers in the context of evolutionary computing, not the exhibition and critique of art in an artistic context.

EA also needs to recognise that aesthetics encompasses much more than the surface appearance of objects and that many other factors and possibilities exist for considering aesthetics in art. Much contemporary art is no longer explicitly concerned with aesthetics (particularly definitions 1, 3, 5 and 6 from Koren's list of Section 3.2). Embracing other understandings opens many new and exciting possibilities for EA beyond the endless generation of self-justified "interesting pictures".

On the other hand - to state the obvious - simply studying current art theory does not necessarily make one a good artist. But good science should necessarily undertake a deep literature review, which would include writing and discourse of art and aesthetics from artists and art theorists. As students, artists always first learn from the history of art, likewise anyone seriously undertaking evolutionary art should know about the history and dialogues of art, and not only from Western culture. Such an approach is the lifetime work of Ellen Dissanayake $[12,13]$, for example. Her work, based on extensive anthropological studies, has been influential on evolutionary theories of human art and the basis for scientific investigation of art and aesthetics [26]. 
Perhaps a more difficult proposition is that EA considers only a narrow and historical concept of art and aesthetics as craft, one that essentially vaguely references Western neo-classical and modernist concepts, but considers art from the limited perspective of objects made by people or machines that can be objectively evaluated. But to concede this, I would argue, is an untenable position for EA research in the long term. Firstly because it privileges one historical and specific understanding of art above all others, which even from a scientific perspective is flawed (why introduce bias into your data without examining the evidence?). Secondly, because even if EA was successful in this limited view of "art", what contribution is it making? It would make little or no contribution to art and the utility of the scientific contribution is also questionable (what hypothesis is it testing and what is it relevant to?).

Another approach would be to concede that, despite its name, EA research is not actually concerned with Art per se, rather it is interested in a scientific study of certain psychological and technical understandings of what human perception is attended to by evolution, and to a lesser extent, by culture and social factors. But a review of papers published over the last five years in EvoMUSART shows this is not the case - the majority of papers focus on the generation of digital "objects" that are considered for their aesthetic (in the EA sense) value and as "art".

Is EA actually more useful in design rather than art? In terms of practical applications, EA has naturally been more successful in design than art, but this is still a relative proposition (it has had very little impact on either). Design also has its own history, critical theory and dialogues and these are rare, if nonexistent, in EvoMUSART proceedings.

A promising, yet to date rarely explored area for EA is for philosophical thought experiments, undertaken by simulation experiments. In this role, EA's goal is not to make art or evolve images for their surface aesthetics. The potential is to illuminate understanding about culture, fads, fashion - even art and the art world itself. These experiments could provide a valuable contribution that is recognised outside of EA, making its agenda and benefit clearer, strengthening the field and improving its impact. Whatever role EA takes, it needs to generate understanding that impacts outside the field itself.

Acknowledgements. The anonymous reviewers provided a number of important insights and comments that assisted in the final version of this paper. This research was supported by an Australian Research Council Discovery Project grant, DP1094064.

\section{References}

1. Arnes, I.: Read_me, run_me, execute_me: Software and its discontents, or: It's the performativity of code, stupid! In: Goriunova, O., Shulgin, A. (eds.) Read_me. Software Art and Cultures Conference, pp. 176-193. University of Århus, Aarhus (2004), http://art.runme.org/1107863582-4268-0/arns.pdf

2. van Assche, C., Moinot, M., Boissier, J.-L.: Images évolutives: Revue virtuelle 5. No. 5, Centre Georges Pompidou (1993) 
3. Ball, P.: Bright earth: the invention of colour. Viking, London (2001)

4. Baumgarten, A.G.: Reflections on Certain Matters Relating to Poetry. Ph.D. thesis (1735)

5. Berlekamp, E.R., Conway, J.H., Guy, R.K.: Winning ways for your mathematical plays, vol. 2. Academic Press, New York (1982)

6. Birkhoff, G.D.: Aesthetic Measure. Harvard University Press, Cambridge (1933)

7. Brafield, P.: Brainstorm: software and habitus in digital design. Digitial Creativity 21(2), 112-126 (2010), doi:10.1080/14626268.2010.483688

8. Colton, S.: The painting fool: Stories from building an automated painter. In: McCormack, J., d'Inverno, M. (eds.) Computers and Creativity, ch.1, pp. 3-38. Springer, Berlin (2012)

9. Dawkins, R.: The Blind Watchmaker. No. 332. Longman Scientific \& Technical, Essex (1986)

10. Dennett, D.C.: Darwin's dangerous idea: evolution and the meanings of life. Simon \& Schuster, New York (1995)

11. Di Paolo, E., Noble, J., Bullock, S.: Simulation models as opaque thought experiments. In: Artifical Life VII (2000)

12. Dissanayake, E.: What is art for? University of Washington Press, Seattle (1988)

13. Dissanayake, E.: Homo aestheticus: where art comes from and why. University of Washington Press, Seattle (1995)

14. Ede, S.: The scientist's mind: The artist's temperament. In: Ede, S. (ed.) Strange and Charmed: Science and the Contemporary Visual Arts, ch.2, pp. 28-49. Calouste Gulbenkian Foundation, London (2000)

15. Foster, H.: The return of the real: the avant-garde at the end of the century. No. xix, 299. MIT Press, Cambridge (1996)

16. Galanter, P.: The Problem with Evolutionary Art Is... In: Di Chio, C., Brabazon, A., Di Caro, G.A., Ebner, M., Farooq, M., Fink, A., Grahl, J., Greenfield, G., Machado, P., O'Neill, M., Tarantino, E., Urquhart, N. (eds.) EvoApplications 2010, Part II. LNCS, vol. 6025, pp. 321-330. Springer, Heidelberg (2010)

17. Gell-Mann, M.: What is complexity? Complexity 1(1), 16-19 (1995)

18. Gerbel, K., Weibel, P. (eds.): Genetic Art - Artificial Life. Ars Electronica, PVS Verleger, Wien, Austria (1993), http://90.146.8.18/en/archives/ festival_archive/festival_catalogs/festival_catalog. asp?iProjectID $=8745$

19. Greenfield, G., Machado, P.: Simulating artist and critic dynamics - an agent-based application of an evolutionary art system. In: Correia, A.D., Rosa, A.C., Madani, K. (eds.) IJCCI, pp. 190-197. INSTICC Press (2009)

20. Humphrey, N.K.: The illusion of beauty. Perception 2, 429-439 (1973)

21. Johnson, C.G.: Fitness in Evolutionary Art and Music: What Has Been Used and What Could Be Used? In: Machado, P., Romero, J., Carballal, A. (eds.) EvoMUSART 2012. LNCS, vol. 7247, pp. 129-140. Springer, Heidelberg (2012)

22. Kant, I.: The critique of judgement. No. 246, 180. Clarendon Press, Oxford (1952)

23. Koren, L.: Which "Aesthetics" Do You Mean?: Ten Definitions. Imperfect Publishing (2010)

24. Krokatsis, H., Walwin, J., Flint, J., Levin, J., O'Reilly, S.: You'll Never Know Drawing and Random Interference. Hayward Gallery, London (2005)

25. Lewis, M.: Evolutionary visual art and design. In: Romero, J., Machado, P. (eds.) The Art of Artificial Evolution: A Handbook on Evolutionary Art and Music, pp. 3-37. Springer (2008)

26. Martindale, C., Locher, P., Petrov, V.M. (eds.): Evolutionary and Neurocognitive Approaches to Aesthetics, Creativity and the Arts. Foundations and Frontiers in Aesthetics. Baywood Publishing Co., Inc. (2007) 
27. McCormack, J.: Open Problems in Evolutionary Music and Art. In: Rothlauf, F., Branke, J., Cagnoni, S., Corne, D.W., Drechsler, R., Jin, Y., Machado, P., Marchiori, E., Romero, J., Smith, G.D., Squillero, G. (eds.) EvoWorkshops 2005. LNCS, vol. 3449, pp. 428-436. Springer, Heidelberg (2005)

28. McCormack, J.: Facing the future: Evolutionary possibilities for human-machine creativity. In: Machado, P., Romero, J. (eds.) The Art of Artificial Evolution: A Handbook on Evolutionary Art and Music, pp. 417-451. Springer (2008)

29. McCormack, J., Bown, O., Dorin, A., McCabe, J., Monro, G., Whitelaw, M.: Ten questions concerning generative computer art. Leonardo to Appear (2012), http://www.csse.monash.edu.au/ jonmc/research/Papers/TenQuestionsLJPreprint.pdf (accepted July 2012)

30. McCormack, J., Dorin, A.: Art, emergence and the computational sublime. In: Dorin, A. (ed.) Second Iteration: Conference on Generative Systems in the Electronic Arts, pp. 67-81. CEMA, Melbourne (2001)

31. Nake, F.: Construction and intuition: Creativity in early computer art. In: McCormack, J., d'Inverno, M. (eds.) Computers and Creativity, pp. 61-94. Springer, Heidelberg (2012)

32. O'Hear, A.: Art and technology: An old tension. Royal Institute of Philosophy Supplement 38, 143-158 (1995)

33. Oreskes, N., Shrader-Frechette, K., Belitz, K.: Verification, validation, and confirmation of numerical models in the earth sciences. Science 263, 641-646 (1994)

34. Parikka, J.: Leonardo book review: The art of artificial evolution: A handbook on evolutionary art and music, http://www.leonardo.info/reviews/nov2008/parikka_art.html

35. Rozenberg, G., Salomaa, A.: Handbook of formal languages. Springer (1996)

36. Saunders, R., Gero, J.S.: Artificial creativity: A synthetic approach to the study of creative behaviour. In: Gero, J.S. (ed.) Proceedings of the Fifth Conference on Computational and Cognitive Models of Creative Design. pp. 113-139. Key Centre of Design Computing and Cognition, Sydney (2001), http://www . arch.usyd.edu. au/ rob/study/publications/ccmcd01/Saunders Gero2001CCMCD.html and http://www.arch.usyd.edu.au/ rob/study/ publications/ccmcd01/SaundersGero2001CCMCD.pdf

37. Schmidhuber, J.: A formal theory of creativity to model the creation of art. In: McCormack, J., d'Inverno, M. (eds.) Computers and Creativity, pp. 323-337. Springer, Heidelberg (2012)

38. Scruton, R.: Beauty. Oxford University Press, Oxford (2009)

39. Takagi, H.: Interactive evolutionary computation: Fusion of the capabilities of EC optimization and human evaluation. Proceedings of the IEEE 89, 1275-1296 (2001)

40. Todd, S., Latham, W.: Mutator: a subjective human interface for evolution of computer sculptures. Tech. rep. (1991)

41. Whitelaw, M.: Metacreation: art and artificial life. MIT Press, Cambridge (2004) 